\title{
Dynamic Behavior of Double-Tapered-Waveguide Distributed Feedback Lasers
}

\author{
S. F. Yu
}

\begin{abstract}
Dynamic behavior of a double-tapered-waveguide (DTWG) distributed feedback (DFB) semiconductor laser is analyzed theoretically. It is found that the relaxation oscillation frequency can be enhanced by the DTWG structure especially for DFB lasers with large coupling-length product.
\end{abstract}

Index Terms - Distributed feedback lasers, double-tapered waveguide, modeling, semiconductor lasers.

\section{INTRODUCTION}

$\mathbf{R}$ ECENTLY, double-tapered-waveguide (DTWG) distributed feedback (DFB) semiconductor lasers with buried heterostructure (BH) is proposed: 1) to reduce the influence of longitudinal spatial hole burning (SHB); 2) to maintain single-mode operation; 3 ) to minimize power density at facets; and 4) to reduce threshold current density [1], [2]. Extensive studies have verified that stable single-mode and high-power operation can be achieved in DTWG-DFB lasers with large coupling-length product $(\kappa L>2.0)$ [2]. However, the relative important characteristics of DTWG-DFB lasers, the dynamic response under direct electrical modulation, has not been considered in their investigation.

In the rate-equation analysis, it has been shown that the square of relaxation oscillation frequency is directly proportional to the magnitude of lateral confinement factor [3]. Furthermore, the damping rate and relaxation oscillation period can be reduced significantly by the combined longitudinal and lateral effects especially for devices with strong lateral optical confinement and large coupling-length product $(k L>2.0)$ [4]. This is due to the induced gain compression [5] and the nonuniform distribution of refractive index profile [6]. Therefore, it is expected that the relaxation oscillation frequency of DTWG-DFB lasers exhibits differently to the uniform waveguide DFB lasers. This is because: 1) the longitudinal distribution of lateral confinement factor as well as 2) the combined longitudinal and lateral SHB effects are dependent on the waveguide dimension (i.e., length and width of the tapered and phase-adjustment region) of DTWG-DFB lasers.

The above paragraph has explained that: 1) the longitudinal variation of lateral confinement factor; 2) the lateral SHB and carrier diffusion; and 3) the longitudinal SHB and refractive index distribution can have influence on the dynamic response of the DTWG-DFB lasers. However, in order to improve the dynamic behavior of the lasers, it is necessary to clarify their

Manuscript received December 16, 1996; revised April 10, 1997. This work was supported by HKU CRCG.

The author is with the Department of Electrical and Electronic Engineering, University of Hong Kong, Pokfulam Road, Hong Kong.

Publisher Item Identifier S 0018-9197(97)05434-1. connection with the relaxation oscillation frequency of the devices and this forms the objective of this paper. In Section II, a self-consistent model including the combined longitudinal and lateral SHB effects as well as carrier diffusion is utilized to study the dynamic behavior of DTWG-DFB lasers. The spatial filtering effects of the tapered waveguide sections are also taken into account by using the effective index method [7]. Using the steady-state approximation, relaxation oscillation frequency of DTWG-DFB lasers is derived analytically. In Section III, the influence of double-tapered geometry on the modulation response of DFB lasers is studied numerically. Finally, a brief conclusion is given in Section IV.

\section{NUMERICAL AND ANALYTICAL ANALYSIS OF DTWG-DFB LASERS}

\section{A. Numerical Model of DTWG-DFB Lasers}

The propagation of forward and reverse fields, $F(z, t)$ and $R(z, t)$, along the laser cavity with tapered waveguide can be described by the modified coupled wave equations as follows:

$$
\begin{aligned}
\left(\frac{1}{v_{g}} \frac{\partial}{\partial t} \pm \frac{\partial}{\partial z}\right)\left[\begin{array}{l}
F \\
R
\end{array}\right]= & {\left[\frac{1}{2}\left(\Gamma_{x} g(z, t)-\alpha_{s}\right)+i \delta \beta(z, t)\right.} \\
& \times\left[\begin{array}{l}
F \\
R
\end{array}\right]+i \kappa(z)\left[\begin{array}{l}
R \\
F
\end{array}\right]
\end{aligned}
$$

where $v_{g}$ is the group velocity, $\Gamma_{x}$ is the transverse confinement factor, and $\kappa$ is the longitudinal coupling coefficient. $\alpha_{s}$ is the absorption and scattering loss in the waveguide and is assumed uniformly distributed along the lateral direction. $g$ is the field gain given by

$$
g(z, t)=\int_{\text {active }} a_{N}\left(N(y, z, t)-N_{o}\right) \psi_{o}^{2}(y) d y
$$

where $N$ is the carrier concentration distribution in the lateral and longitudinal directions, $a_{N}$ is the differential gain, and $\psi_{o}$ is the lateral field distribution. The deviation from the Bragg condition $\delta \beta$ is given as

$$
\delta \beta(z, t)=\frac{2 \pi}{\lambda_{\circ}} n_{\mathrm{eff}}(z, t)-\frac{\pi}{\Lambda}
$$

where $c$ is the speed of light and $\Lambda$ is the pitch of the grating. $n_{\mathrm{eff}}$ is the effective refractive index in the longitudinal direction. The change of effective refractive index can be calculated by using the method given in [7] and [8]. 
The time-dependent rate equation of carrier concentration is given by

$$
\begin{aligned}
\frac{d N(y, z, t)}{d t}= & \frac{J(y, z, t)}{q d}-\frac{N(y, z, t)}{\tau}-v_{g} a_{N} \\
& \times\left(N(y, z, t)-N_{o}\right) \mathrm{P}(y, z, t) \\
& +D_{f} \frac{\partial^{2} N(y, z, t)}{\partial y^{2}}
\end{aligned}
$$

where $J$ is the injection current density profile, $d$ is the thickness of the active layer, $D_{f}$ is the diffusion constant, $\tau$ is the carrier lifetime, and $q$ is the electron charge. The photon density distribution $\mathbf{P}$ is given as

$$
\begin{aligned}
\mathbf{P}(y, z, t) & =\left[|F(z, t)|^{2}+|R(z, t)|^{2}\right] \psi_{o}^{2}(y) \\
& =P(z, t) \psi_{o}^{2}(y) .
\end{aligned}
$$

To simplify our calculation, the inhomogeneous carrier distribution in the lateral direction is expanded in a Fourier series. The first-order approximation of carrier distribution is then given by [9]

$$
N(y, z, t)=N_{p}(z, t)-N_{1}(z, t) \cos (2 \pi y / w)
$$

where $w$ is the width of the waveguide, $N_{p}$ is the average carrier distribution in the longitudinal direction, and $N_{1}$ is the perturbation of carrier concentration in the lateral direction. Substituting (6) into (2) and (4), with $J$ assumed constant across the active layer and integrating (2) and (4) over the active layer, including the gain compression coefficient $\varepsilon$, we obtain the modified field gain expression given as

$$
g(z, t)=\frac{a_{N}\left\{\Gamma_{y}(z)\left(N_{p}(z, t)-N_{o}\right)-\xi_{1}(z) N_{1}(z, t)\right\}}{(1+\varepsilon P(z, t))}
$$

and the rate equations of carrier densities given as

$$
\begin{aligned}
\frac{d N_{p}(z, t)}{d t}= & \frac{J(z, t)}{q d}-\frac{N_{p}(z, t)}{\tau} \\
& -\frac{v_{g} a_{N}\left\{\Gamma_{y}(z)\left(N_{p}(z, t)-N_{o}\right)-\xi_{1}(z) N_{1}(z, t)\right\}}{(1+\varepsilon P(z, t))} \\
& \times P(z, t) \quad(7 \mathrm{~b}) \\
\frac{d N_{1}(z, t)}{d t}= & \frac{2 v_{g} a_{N}\left\{\xi_{1}(z)\left(N_{p}(z, t)-N_{o}\right)-\xi_{2}(z) N_{1}(z, t)\right\}}{(1+\varepsilon P(z, t))} \\
& \times P(z, t)-\frac{1+\gamma}{\tau} N_{1}(z, t)
\end{aligned}
$$

where $\Gamma_{y}$ is the confinement factor in the lateral direction and $\xi_{1}$ and $\xi_{2}$ are the first- and second-order coupling parameters of the carriers and the optical field $\psi_{o}$. Their definitions are given in Appendix A. The parameter $\gamma$ is given by

$$
\gamma=4 \pi^{2} \tau D_{f} / w^{2} \text {. }
$$

A self-consistent large-signal calculation can be obtained by solving (1) and (7) simultaneously.

It must be noted that the round-trip conditions of DTWGDFB lasers are affected by their waveguide dimension. This is because the longitudinal propagation constant is changed according to the stripe width of waveguide, such that the deviation in propagation constant from Bragg constant is also dependent on the waveguide dimension. Therefore, the values of $\Gamma_{y}, \kappa, \delta \beta, \xi_{1}$, and $\xi_{2}$ are dependent on the stripe width of the waveguide and can be evaluated by the effective index method [7]. Detailed calculation of these parameters can be found in Appendix A.

\section{B. Relaxation Oscillation Frequency of DTWG-DFB Lasers: Analytical Analysis}

It is believed that the lateral and longitudinal effects (including waveguide structure and SHB profile) will have effect on the relaxation oscillation frequency of DTWG-DFB lasers. In order to investigate the influence of lateral and longitudinal effects, the analytical expression of relaxation oscillation frequency is derived from the time-dependent rate equations of photon density and carrier concentration. Therefore, the requirement for the enhancement of relaxation oscillation frequency of DTWG-DFB lasers can be clarified.

The derivation is started from the coupled wave equations. The rate equation of photon density can be deduced by multiplying (1) with its complex conjugate of the forward and reverse propagation fields and adding the two equations and their conjugates to obtain

$$
\frac{\partial P}{\partial t}=\left(\Gamma_{x}\left(\Gamma_{y} G_{P}\left(N_{P}\right)-v_{g} a_{N} \xi_{1} N_{1}\right)-v_{g} \alpha_{p}\right) P
$$

where $P=|F|^{2}+|R|^{2}, \alpha_{p}=\alpha_{s}+\left(\partial|F|^{2}+|R|^{2} / \partial z\right) /$ $\left(|F|^{2}+|R|^{2}\right)$ and $G_{P}\left(N_{P}\right)=v_{g} a_{N}\left(N_{P}-N_{o}\right)$.

Rewriting (7b) and (7c) in terms of $G_{p}\left(N_{P}\right)$, we obtain

$$
\begin{aligned}
& \frac{d N_{p}}{d t}=\frac{J}{q d}-\frac{N_{p}}{\tau}-\left\{\Gamma_{y} G_{P}\left(N_{P}\right)-v_{g} a_{N} \xi_{1} N_{1}\right\} P \\
& \frac{d N_{1}}{d t}=2\left\{\xi_{1} G_{P}\left(N_{P}\right)-v_{g} a_{N} \xi_{2} N_{1}\right\} P-\frac{1+\gamma}{\tau} N_{1} .
\end{aligned}
$$

In the derivation of (9)-(11), gain compression has been ignored. In (11), if the frequency of the modulation signal is less than the effective electron lifetime $\tau /(1+\gamma)$, it is possible to obtain an approximate solution to $N_{1}$ by setting the derivative to zero [9]. We have

$$
\begin{aligned}
N_{1}(z, t) & =\tau^{\prime} \xi_{1} G_{P}\left(N_{P}\right) P /\left(1+\varepsilon_{2} P\right) \\
& \approx \tau^{\prime} \xi_{1} G_{P}\left(N_{P}\right)\left(1-\varepsilon_{2} P\right) P
\end{aligned}
$$

where $\tau^{\prime}=2 \tau /(1+\gamma)$ and $\varepsilon_{2}=\tau^{\prime} v_{g} a_{N} \xi_{2}$. The rate equations can be simplified by substituting (12) into (9) and (10). We have

$$
\begin{aligned}
\frac{\partial P}{\partial t}= & \left(\Gamma _ { x } \Gamma _ { y } G _ { P } ( N _ { P } ) \left(1-\left(\varepsilon_{1}^{2} / \Gamma_{y}\right) P\right.\right. \\
& \left.\left.+\left(\varepsilon_{1}^{2} \varepsilon_{2} / \Gamma_{y}\right) P^{2}\right)-v_{g} \alpha_{p}\right) P \\
\frac{d N_{p}}{d t}= & \frac{J}{q d}-\frac{N_{p}}{\tau}-\Gamma_{y} G_{P}\left(N_{P}\right)\left(1-\left(\varepsilon_{1}^{2} / \Gamma_{y}\right) P\right. \\
& \left.+\left(\varepsilon_{1}^{2} \varepsilon_{2} / \Gamma_{y}\right) P^{2}\right) P
\end{aligned}
$$

where $\varepsilon_{1}^{2}=\tau^{\prime} v_{g} a_{N} \xi_{1}^{2} \cdot \varepsilon_{1}$ and $\varepsilon_{2}$ can be considered to be the effective gain compression factors arisen from the lateral effects.

We separate the dynamic average photon density and carrier concentration by writing the local photon density and carrier 
concentration as

$$
\begin{aligned}
P(z, t) & =\bar{P}(t)(1+f(z)) \\
N_{P}(z, t) & =N_{P}(t)+\delta N(t) f(z)
\end{aligned}
$$

where $\delta N \ll N_{P}, f(z)$ being a function that is smaller than unity and for which

$$
\int_{0}^{L} f(z) d z=0
$$

where $L$ is the cavity length. Substituting (14) into (13) and ignoring the effective gain compression factor in (13), as the gain saturation parameters $\varepsilon_{1}$ and $\varepsilon_{2}$ due to the lateral effects have less influence on the damping rate of lasers, we obtain

$$
\begin{aligned}
\frac{d \bar{P}}{d t} & =\left(\Gamma_{x}\left(\Gamma_{y 1} G_{P}\left(N_{P}\right)+\Gamma_{y 2} v_{g} a_{N} \delta N\right)-v_{g} \alpha_{p}^{\prime}\right) \bar{P} \\
\frac{d N_{p}}{d t} & =\frac{J}{q d}-\frac{N_{p}}{\tau}-\left(\Gamma_{y 1} G_{P}\left(N_{P}\right)+\Gamma_{y 2} v_{g} a_{N} \delta N\right) \bar{P} .
\end{aligned}
$$

$\delta N$ can be approximated by steady-state consideration [5] and is given by

$$
\delta N=-\tau v_{g} a_{N} \Gamma_{y 2} \bar{P} / \eta\left(1+\chi_{3} \bar{P}\right)
$$

where $\chi_{3}=\tau v_{g} a_{N} \Gamma_{y 3} / \eta, \Gamma_{y 1}\left(=\bar{\Gamma}_{y}+\Gamma_{L p}\right), \Gamma_{y 2}$, and $\Gamma_{y 3}$ are defined as first-, second-, and third-order lateral confinement factors, $\alpha_{p}^{\prime}\left(=\alpha_{s}+\alpha_{L p}\right)$ is the equivalent cavity loss, and $\eta$ is a normalizing factor. Their definitions are given in Appendix B.

Using small-signal analysis technique, the relaxation oscillation frequency $\omega_{f}$ of the DTWG-DFB lasers can be deduced and is given by

$$
\begin{aligned}
\omega_{f}^{2} & =v_{g}^{2} a_{N} \Gamma_{y 1} \bar{P}_{0} \alpha_{p}^{\prime}\left(1+2\left(\chi_{3}-\chi_{4}\right) \bar{P}_{0}-3 \chi_{3} \chi_{4} \bar{P}_{0}^{2}\right) \\
& \approx v_{g}^{2} a_{N} \Gamma_{y 1} \bar{P}_{0} \alpha_{p}^{\prime}
\end{aligned}
$$

where $\bar{P}_{0}$ is the steady-state photon density and $\chi_{4}=\chi_{3}+$ $\tau v_{g} a_{N} \Gamma_{y 2}^{2} / \Gamma_{y 1} \eta$. The influence of gain saturation due to longitudinal SHB of carrier concentration (i.e., $\chi_{3}$ and $\chi_{4}$ ) is ignored in (18).

In the derivation of (18), the influence of gain compression due to combined lateral and longitudinal effects has been ignored and this assumption will be verified in the following numerical analysis. On the other hand, the simplified expression of $\omega_{f}$ shows some important insights into how the tapered waveguide structure influences the relaxation oscillation frequency of DTWG-DFB lasers. As we can see, the first-order lateral confinement factor $\Gamma_{y 1}$ is defined as the sum of $\bar{\Gamma}_{y}$ (i.e., average value of $\Gamma_{y}$ ) and $\Gamma_{L p}$ (i.e., the overlapping integral of $f(z)$ and $\Gamma_{y}$ ). The equivalent cavity loss $\alpha_{p}^{\prime}$ is equal to the sum of $\alpha_{s}$ (i.e., cavity loss) and $\alpha_{L p}$ (i.e., the overlapping integral of $\alpha_{p}(z)$ and $f(z)$ ) (see Appendix B). These indicate that the terms $\bar{\Gamma}_{y}, \Gamma_{L p}, \alpha_{s}$, and $\alpha_{L p}$ should be optimized in order to enhance the value of $\omega_{f}$.

The possibilities to maximize the value of $\omega_{f}$ are discussed as follows.

1) In order to maximize the value of $\bar{\Gamma}_{y}$, a wide stripe-width design should be adopted provided that the suppression of high-order lateral modes can be maintained by the diffraction effects of the DTWG structure.
2) It is assumed that $\alpha_{s}$ is uniform along the active and blocking regions such that its magnitude will not be affected by the laser structure.

3) A positive value of $\Gamma_{L p}$ with maximum magnitude can be obtained if the longitudinal distribution of $\Gamma_{y}$ and the function $f(z)$ are matched. It must be noted that the longitudinal profile of $\Gamma_{y}$ is mainly determined by the waveguide design; however, the longitudinal distribution of $f(z)$ is affected by the waveguide design as well as the $\kappa L$ of the lasers. In order to maximize the value of $\Gamma_{L p}$, the profile of $f(z)$ should be selected to match with $\Gamma_{y}$.

4) In order to maximize the overlapping integral $\alpha_{L p}$, it is required to study the relation between $\partial|F|^{2}-$ $|R|^{2} / \partial z(\equiv q(z))$ and $f(z)$. It is expected that the longitudinal distribution function $q(z)$ is affected by the waveguide design as well as the $\kappa L$ of the lasers. On the other hand, for devices with symmetric waveguide structure, it can be shown that the function $q(z)$ has a symmetric distribution profile and its shape is close to the longitudinal distribution of carrier concentration. Let's consider the laser with symmetric waveguide structure such that we can write

$$
|F(z)|^{2}+|R(z)|^{2}=|F(z)|^{2}+|F(L-z)|^{2}
$$

and let $\partial|F(z)|^{2} / \partial z=|G(z)|^{2}$. Then the function $q(z)$ can be rewritten as

$$
\begin{aligned}
q(z) & =\partial|F(z)|^{2}-|R(z)|^{2} / \partial z \\
& =\partial|F(z)|^{2}-|F(L-z)|^{2} / \partial z \\
& =|G(z)|^{2}+|G(z-L)|^{2} .
\end{aligned}
$$

Hence, the longitudinal profile of $q(z)$ is inversely proportional to the longitudinal distribution of photon density (as well as $f(z)$ ) due to the partial differentiation terms. Therefore, the overlapping integral of $\alpha_{L p}$ may give a negative value and is minimized for high $\kappa L$ devices because high $\kappa L$ devices accumulate more photon energy inside the cavity than low $\kappa L$ devices (i.e., severe longitudinal SHB for high $\kappa L$ devices).

5) It has been shown that high $\kappa L$ devices have relatively high $\omega_{f}$. This is because the total energy stored inside the laser cavity, which is proportional to $\omega_{f}$, is high for high $\kappa L$ devices [4]. Therefore, we expect $\omega_{f}$ of DTWG-DFB lasers increases with $\kappa L$.

From the above prediction on the dynamic behavior of DTWG-DFB lasers, it is shown that $\omega_{f}$ in general increases with stripe width and $\kappa L$; however, the variation of $\Gamma_{L p}$ and $\alpha_{L p}$ may work against the change of $\omega_{f}$. In the following numerical analysis, we study the influence of stripe width and $\kappa L$ on the variation of $\omega_{f}$ and the overlapping integrals (i.e., $\Gamma_{L p}$ and $\left.\alpha_{L p}\right)$ separately.

\section{Simulation Results}

\section{A. DTWG-DFB Lasers: Structure and Material Composition}

The waveguide structure and the material composition of the lasers used in this paper are similar to that given in [2]. 


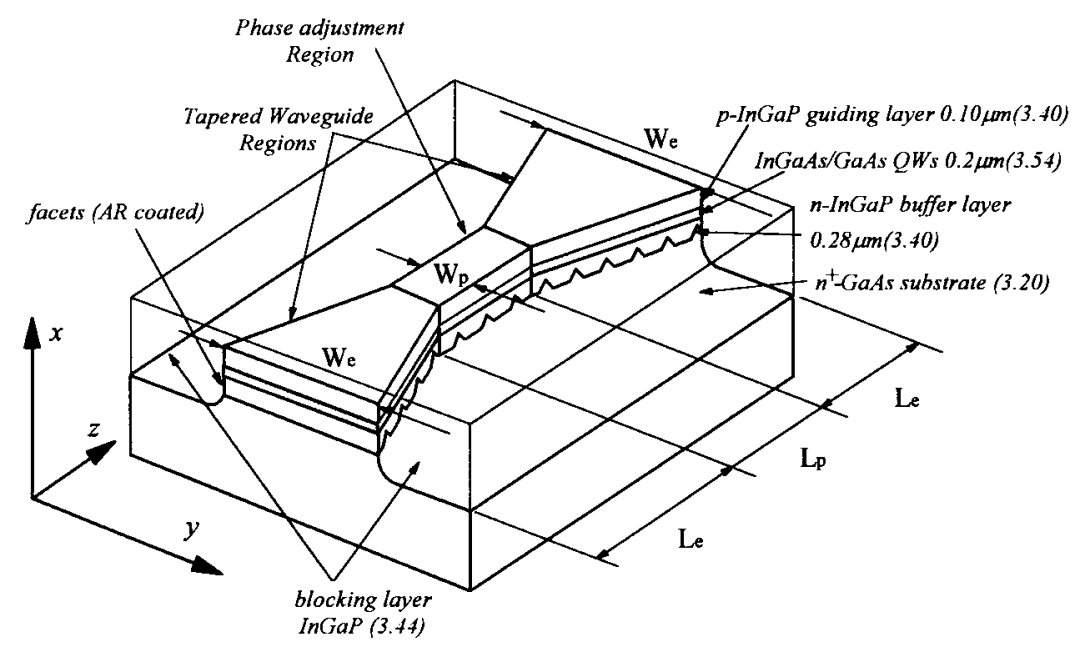

Fig. 1. Schematic of a buried structure DFB laser with double-tapered-waveguide structure.

TABLE I

Device Parameters Used in the Model

\begin{tabular}{l|l}
\hline Parameters (symbol) & Magnitude \\
\hline \hline Carrier lifetime $(\tau)$ & $3 \times 10^{-9} \mathrm{~s}$ \\
\hline Differential gain $\left(\mathrm{a}_{\mathrm{N}}\right)$ & $3 \times 10^{-16} \mathrm{~cm}^{2}$ \\
\hline Transparency carrier density $\left(\mathrm{N}_{\mathrm{o}}\right)$ & $1.5 \times 10^{18} \mathrm{~cm}^{-3}$ \\
\hline Linewidth enhancement factor $\left(\alpha_{\mathrm{m}}\right)$ & 3.0 \\
\hline Absorption and scattering loss $\left(\alpha_{\mathrm{s}}\right)$ & $40 \mathrm{~cm}^{-1}$ \\
\hline Effective group refractive index $\left(\mathrm{n}_{\mathrm{g}}\right)$ & 3.70 \\
\hline Thickness of the active layer $(\mathrm{d})$ & $0.2 \mu \mathrm{m}$ \\
\hline Approximate emission wavelength $\left(\lambda_{\mathrm{o}}\right)$ & $0.98 \mu \mathrm{m}$ \\
\hline Transverse optical confinement factor $\left(\Gamma_{\mathrm{x}}\right)$ & 0.35 \\
\hline Period of grating $(\Lambda)$ & $0.145 \mu \mathrm{m}$ \\
\hline Group velocity $\left(v_{\mathrm{g}}\right)$ & $8 \times 10^{9} \mathrm{~cm} / \mathrm{s}$ \\
\hline magnitude of Left facet reflectivity $\left(\left|\mathrm{r}_{\mathrm{L}}\right|\right)$ & 0 \\
\hline magnitude of Right facet reflectivity $\left(\left|\mathrm{r}_{\mathrm{R}}\right|\right)$ & 0 \\
\hline
\end{tabular}

It is assumed that the waveguide geometry has a doubletapered structure with a phase-adjustment region (PAR) in between (see Fig. 1). The laser is composed of five layers of semiconductor materials: the $\mathrm{p}^{+}-\mathrm{GaAs}$ cladding layer, $\mathrm{p}$ InGaP guiding layer, the InGaAs-GaAs $(0.98 \mu \mathrm{m})$ quantum well's active region, the $\mathrm{n}-\mathrm{InGaP}$ buffer layer and $\mathrm{n}^{+}$-GaAs substrate. The BH is used to provide strong optical and electrical confinement along the lateral direction. Device parameters used in the model can be found in Table I.

In the following sections, it is assumed that the lasers have symmetric waveguide geometry with both facets antireflectioncoated. The width of the PAR $W_{p}$ and the tapered waveguide near facets $W_{e}$ varies from 1 to $2 \mu \mathrm{m}$ and from 2 to $6 \mu \mathrm{m}$, respectively. The corresponding length of the tapered section $L_{e}$ is equal to $135 \mu \mathrm{m}$ and that of PAR $L_{p}$ is equal to 110 $\mu \mathrm{m}$ such that single-mode operation can be maintained. For the reason of comparison, the steady-state photon density at the facets is set to be around $2 \times 10^{14} \mathrm{~cm}^{-3}$.

\section{B. Relaxation Oscillation Frequency of DTWG-DFB Lasers: Numerical Analysis}

In the numerical analysis of BH-DFB lasers given in [4], it is shown that the magnitude of $\omega_{f}$ is affected by the magnitude of $\Gamma_{y}, \xi_{1}$, and $\xi_{2}$, especially for $\Gamma_{y}, \xi_{1}$, and $\xi_{2}$ approach their

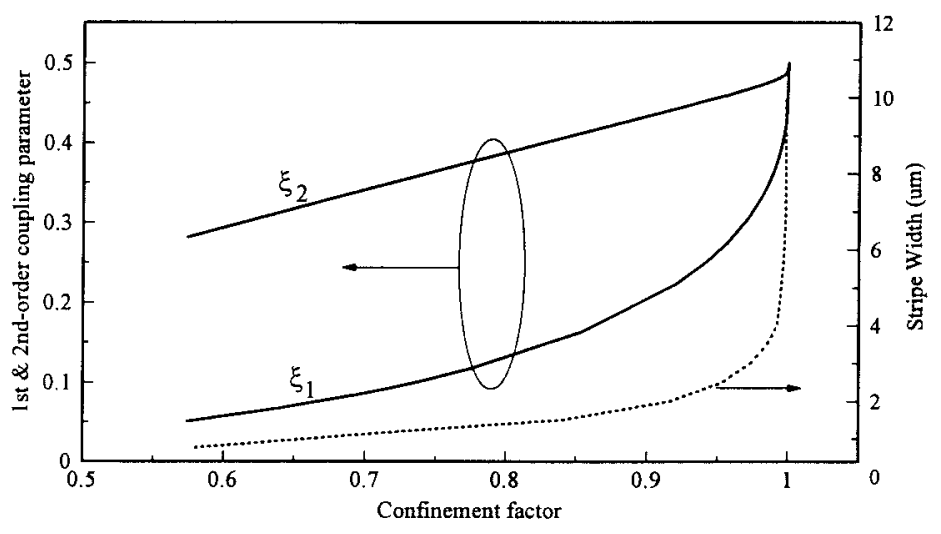

Fig. 2. The variation of stripe width, first-order $\xi_{1}$ and second-order $\xi_{2}$ coupling parameters with the lateral confinement factor $\Gamma_{y}$.

limited values (i.e., $\Gamma_{y} \rightarrow 1.00, \xi_{1} \rightarrow 0.50, \xi_{2} \rightarrow 0.50$ ). In DTWG-DFB lasers, these parameters are varied along the laser waveguide. Fig. 2 shows the general plot of $\xi_{1}$ and $\xi_{2}$ against $\Gamma_{y}$ for a strong index-guided waveguide. The corresponding variation of stripe width with $\Gamma_{y}$ is also shown in the diagram. As we can see for the stripe width larger than or equal to $4 \mu \mathrm{m}$, the magnitude of $\Gamma_{y}, \xi_{1}$, and $\xi_{2}$ approach their limited values. In the following paragraphs, the influence of $\Gamma_{y}, \xi_{1}$, and $\xi_{2}$ (due to the longitudinal variation of stripe width) on the dynamic behavior of DTWG-DFB lasers is analyzed numerically.

Fig. 3 shows the variation of $\omega_{f}^{2}$ as a function of $W_{e}$ for devices with $D_{f}=5 \mathrm{~cm}^{2} \mathrm{~s}^{-1}$ and $\kappa_{o}=30,50$, and $80 \mathrm{~cm}^{-1}$. As we can see, $\omega_{f}^{2}$ increases with $W_{e}$ and is high for the device with $W_{p}=2 \mu \mathrm{m}$ and $\kappa_{o}=80 \mathrm{~cm}^{-1}$. The influence of $W_{e}$ on the transient response of the output power for device with $W_{p}=2 \mu \mathrm{m}, D_{f}=5 \mathrm{~cm}^{2} \mathrm{~s}^{-1}$ and $\kappa_{o}=50 \mathrm{~cm}^{-1}$ is shown in Fig. 4. The damping rate for devices with $W_{e}=2$ and $5 \mu \mathrm{m}$ exhibit similar magnitude such that our assumption used in the derivation of (16) (i.e., ignorance of gain compression due to lateral effects) is satisfied.

The influence of $D_{f}$ on $\omega_{f}^{2}$ of DTWG-DFB lasers with $\kappa_{o}=$ $50 \mathrm{~cm}^{-1}$ is shown in Fig. 5. As we can see, $\omega_{f}^{2}$ increases with $D_{f}$ from 0 to $5 \mathrm{~cm}^{2} \cdot \mathrm{s}^{-1}$ and is saturated for $D_{f} \geq 5 \mathrm{~cm}^{2} \cdot \mathrm{s}^{-1}$. 


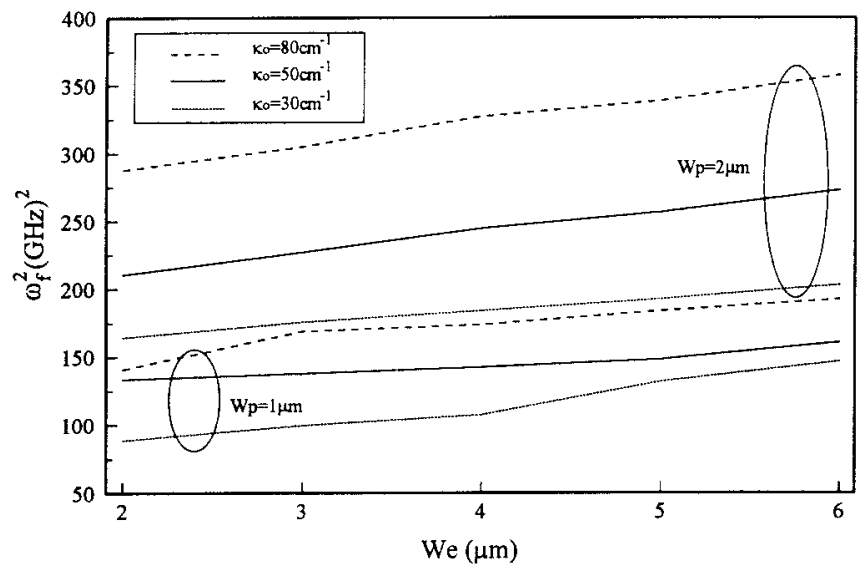

Fig. 3. The variation of $\omega_{f}^{2}$ with $W_{e}$ for lasers with $D_{f}=5 \mathrm{~cm}^{2} \cdot \mathrm{s}^{-1}$, $W_{p}=1$ and $2 \mu \mathrm{m}, \kappa_{o}=30 \mathrm{~cm}^{-1}$ (dotted line), $\kappa_{o}=50 \mathrm{~cm}^{-1}$ (solid line) and $\kappa_{0}=80 \mathrm{~cm}^{-1}$ (dashed line).

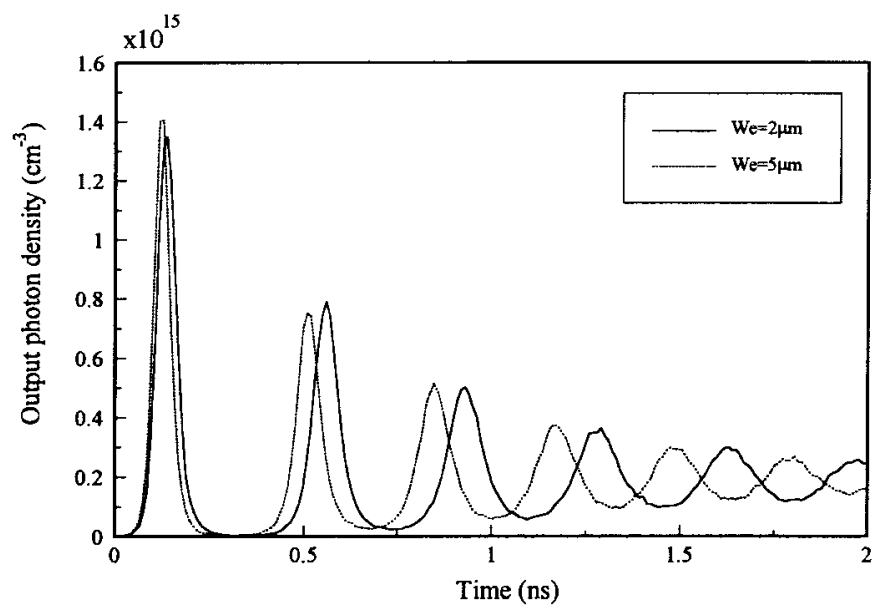

Fig. 4. Transient response of the output photon density for devices with $W_{e}=2$ and $5 \mu \mathrm{m}$. The other parameters are $D_{f}=5 \mathrm{~cm}^{2} \cdot \mathrm{s}^{-1}, \kappa_{o}=50$ $\mathrm{cm}^{-1}$ and $W_{p}=2 \mu \mathrm{m}$.

The magnitude of $\omega_{f}^{2}$ remains unchanged for further increase of $D_{f}$ to $50 \mathrm{~cm}^{2} \cdot \mathrm{s}^{-1}$. The influence of $D_{f}$ on the transient response of the output power for devices with $W_{p}=2 \mu \mathrm{m}$, $W_{e}=5 \mathrm{~cm}^{2} \cdot \mathrm{s}^{-1}$ and $\kappa_{o}=50 \mathrm{~cm}^{-1}$ is also shown in Fig. 6 . As we can see, the lateral carrier diffusion has less influence on the damping rate of the devices.

The dynamic behavior of DTWG-DFB lasers can be summarized as follows.

1) $\omega_{f}$ increases with $W_{e}$ and $W_{p}$. This is because the increase of $W_{e}$ and $W_{p}$ increases the value of $\Gamma_{y}, \xi_{1}$, and $\xi_{2}$ (i.e., this is equivalent to increasing the value of $\left.\bar{\Gamma}_{y}\right)$.

2) $\omega_{f}$ is saturated at a high magnitude for $D_{f} \geq 5 \mathrm{~cm}^{2} \mathrm{~s}^{-1}$ and this characteristic is different to uniform-stripe $\mathrm{BH}$ DFB lasers [4].

3) $W_{e}, W_{p}$, and $D_{f}$ have less influence on the damping rate of DTWG-DFB lasers and this implies that the induced gain compression due to lateral effects can be ignored in our analysis.

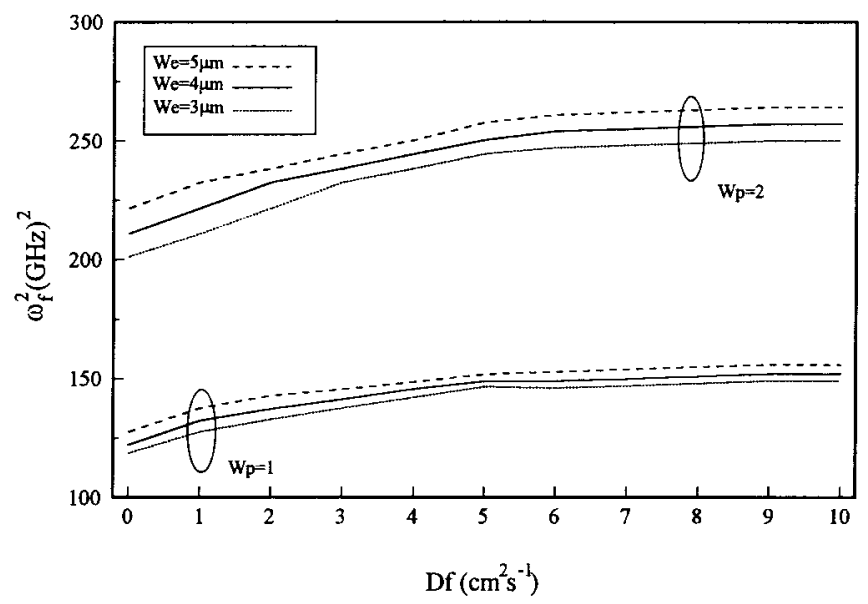

Fig. 5. The variation of $\omega_{f}^{2}$ with $D_{f}$ for lasers with $\kappa_{o}=50 \mathrm{~cm}^{-1}, W_{p}=$ 1 and $2 \mu \mathrm{m}, W_{e}=3 \mu \mathrm{m}$ (dotted line), $W_{e}=4 \mu \mathrm{m}$ (solid line), and $W_{e}=$ $5 \mu \mathrm{m}$ (dashed line).

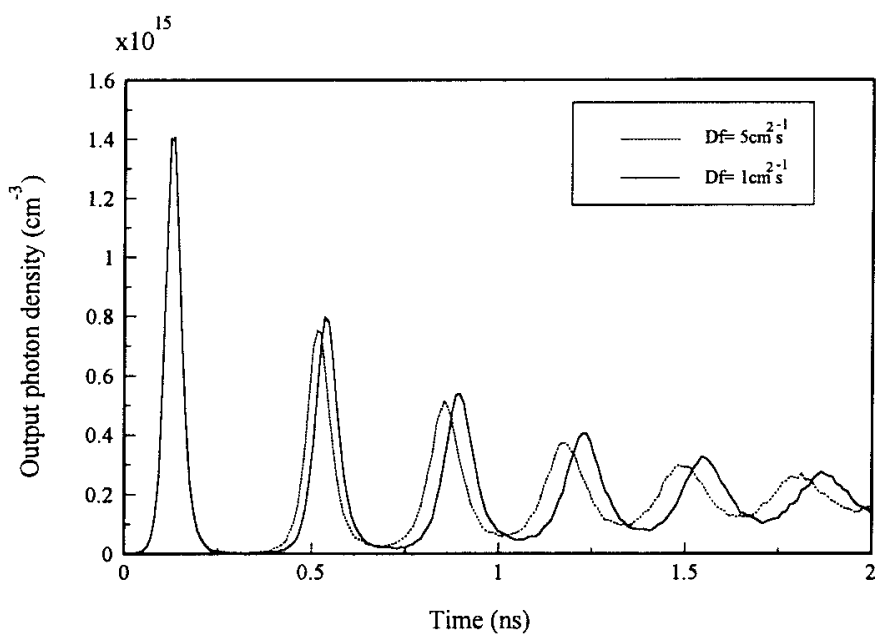

Fig. 6. Transient response of the output optical photon density for devices with $D_{f}=1$ and $5 \mathrm{~cm}^{2} \cdot \mathrm{s}^{-1}$. The other parameters are $W_{e}=5 \mu \mathrm{m}, \kappa_{o}=$ $50 \mathrm{~cm}^{-1}$, and $W_{p}=2 \mu \mathrm{m}$.

4) $\omega_{f}$ increases with $\kappa_{O}$; however, $\kappa_{O}$ has less influence on the damping rate of the lasers. This indicates that the induced gain compression due to longitudinal SHB has less contribution on the dynamic behavior of the devices.

The numerical results show that $\omega_{f}$ increases with stripe width (i.e., $W_{e}$ and $W_{p}$ ) and $\kappa L$, which partly verifies our analytical prediction on the dynamic behavior of DTWG-DFB lasers. Now, we further investigate the relation between the overlapping integrals (i.e., $\Gamma_{L p}$ and $\alpha_{L p}$ ) and the devices' parameters (i.e., $W_{e}, W_{p}$, and $\kappa L$ ).

It is assumed in this study that devices have $W_{p}=2 \mu \mathrm{m}$ and $D_{f}=5 \mathrm{~cm}^{2} \cdot \mathrm{s}^{-1}$. As shown in Fig. 7, $\Gamma_{y}$ exhibits a "U" shape with minimum at the PAR and maximum near the facets. $\Gamma_{y}$ approaches its limited value (i.e., $\Gamma_{y} \approx 1.00$ near the facets region) as $W_{e}$ approaches $4 \mu \mathrm{m}$. The function $f(z)$ defined in (14) can be obtained from the longitudinal distribution of optical density

$$
f(z)=\left(|F(z)|^{2}+|R(z)|^{2}\right) / \bar{P}_{0}-1 .
$$




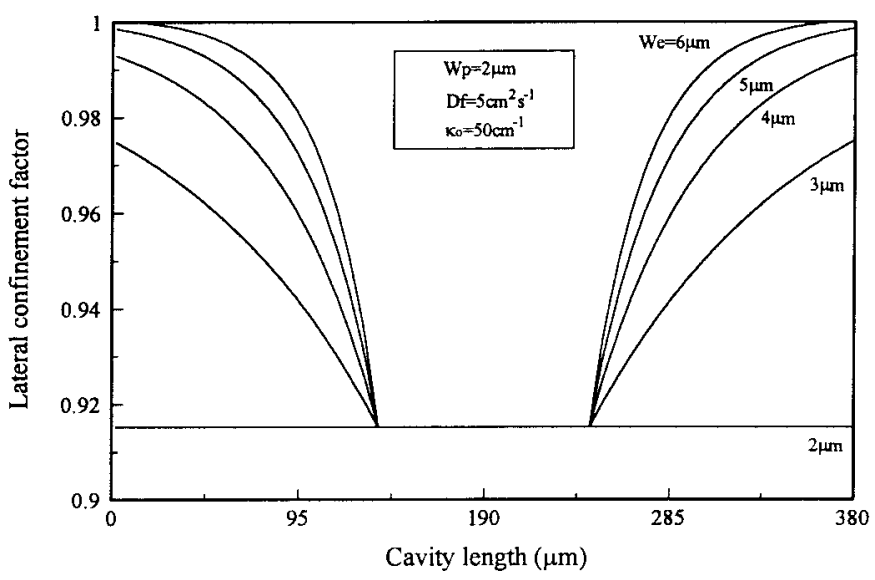

Fig. 7. The variation longitudinal distribution of lateral confinement factor $\Gamma_{y}$ with $W_{e}$. The other parameters are $D_{f}=5 \mathrm{~cm}^{2} \cdot \mathrm{s}^{-1}, \kappa_{o}=50 \mathrm{~cm}^{-1}$, and $W_{p}=2 \mu \mathrm{m}$.

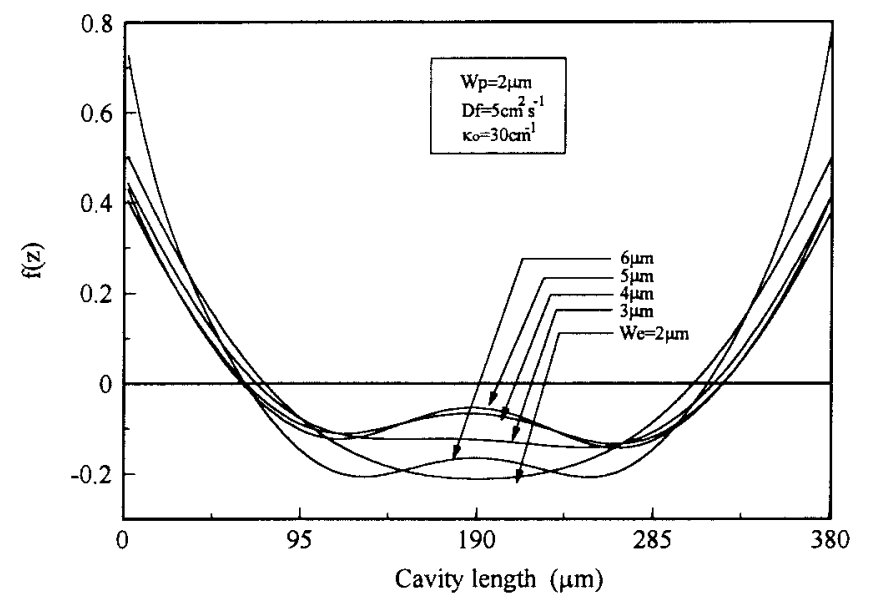

(a)

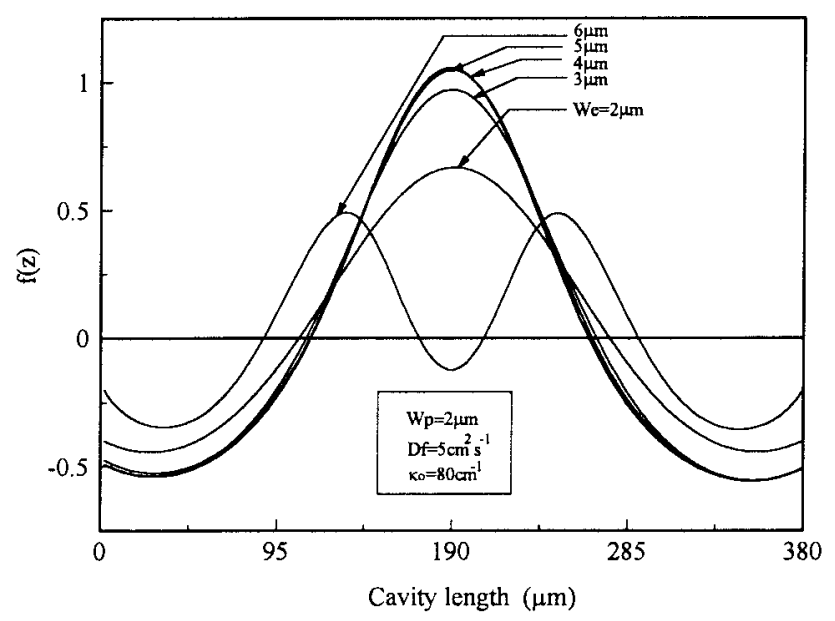

(b)

Fig. 8. The variation of $f(z)$ with $W_{e}$ for devices with (a) $\kappa_{o}=30 \mathrm{~cm}^{-1}$ and (b) $\kappa_{o}=80 \mathrm{~cm}^{-1}$. The other parameters are $D_{f}=5 \mathrm{~cm}^{2} \cdot \mathrm{s}^{-1}$ and $W_{p}=2 \mu \mathrm{m}$.

Fig. 8 plots the longitudinal distribution of $f(z)$ for devices with $\kappa_{O}=30$ and $80 \mathrm{~cm}^{-1}$ for a range of $W_{e}$. As we can see, the profile of $f(z)$ is close to $\Gamma_{y}$ for a device with $\kappa_{O}=30 \mathrm{~cm}^{-1}$ but is convex upwards near the PAR for a device with $\kappa_{O} \geq 50 \mathrm{~cm}^{-1}$ which is totally mismatched with

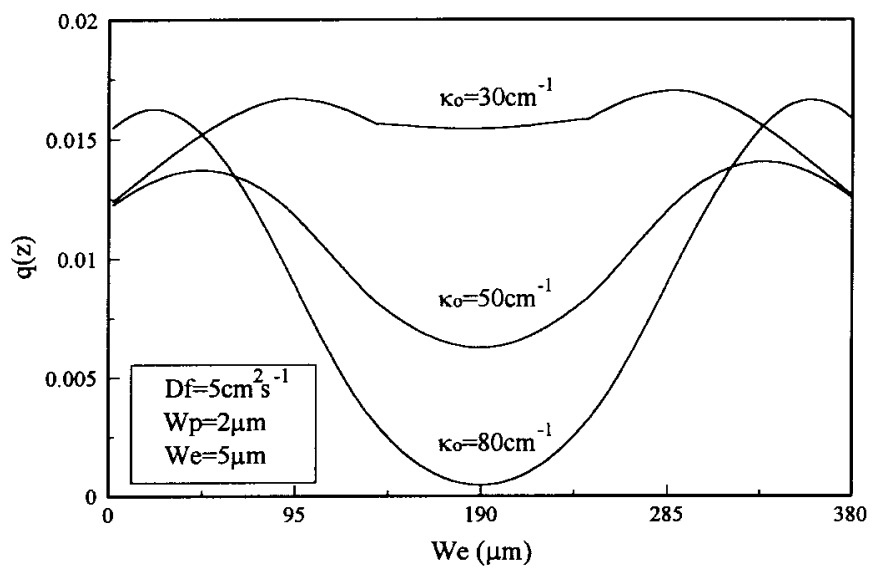

Fig. 9. The longitudinal variation of $q(z)$ with $\kappa_{o}$. The other parameters are $D_{f}=5 \mathrm{~cm}^{2} \cdot \mathrm{s}^{-1}, W_{e}=5 \mu \mathrm{m}$, and $W_{p}=2 \mu \mathrm{m}$.

the profile of $\Gamma_{y}$. Therefore, it is expected that the value of $\Gamma_{L p}$ can be enhanced for $\kappa_{O} \leq 30 \mathrm{~cm}^{-1}$ due to matching of the longitudinal profile of $f(z)$ and $\Gamma_{y}(z)$. The longitudinal distribution of $q(z)$ for $W_{e}=5 \mu \mathrm{m}$ is also shown in Fig. 9. It is shown that the longitudinal distribution of $q(z)$ is inversely proportional to the profile of $f(z)$. The corresponding variation of $\Gamma_{L p}$ and $\alpha_{L p}$ with $W_{e}$ is shown in Fig. 10. As we expected, the value of $\Gamma_{L p}$ and $\alpha_{L p}$ decreases with the increase of $W_{e}$ for devices with $\kappa_{O} \geq 50 \mathrm{~cm}^{-1}$. This is because: 1) the "U" shape profile of $\Gamma_{y}$ is mismatched with the $f(z)$ which is convex upwards near the PAR and hence the value of $\Gamma_{L p}$ is reduced; 2) $f(z)$ is more convex upwards with the increase of $W_{e}$ and $\kappa_{O}$ such that the value of $\alpha_{L p}$ is reduced. We can conclude that $\Gamma_{L p}$ and $\alpha_{L p}$ are reduced with the increase of $W_{e}$ and $\kappa_{o}$ due to the longitudinal SHB which may reduce the value of $\omega_{f}$. However, from our numerical study on $\omega_{f}$, it is shown that $\omega_{f}$ increases with $W_{e}$ and $\kappa_{o}$ which indicates that the effects of $\Gamma_{L p}$ and $\alpha_{L p}$ have been overcome by $\bar{\Gamma}_{y}$ and total energy stored inside the laser cavity.

\section{CONCLUSION}

The influence of a DTWG structure on $\omega_{f}$ of DFB lasers is clarified. By using the first-order approximation on the combined lateral and longitudinal distribution of carrier concentration, the $\omega_{f}$ of DTWG-DFB lasers is deduced analytically. It is illustrated from (18) that the longitudinal SHB reduces $\omega_{f}$ through the terms $\Gamma_{L p}$ and $\alpha_{L p} . \Gamma_{L p}$ is arisen from the DTWG structure and $\alpha_{L p}$ is an inherent property of DFB lasers. However, $\omega_{f}$ is an increasing function of stripe width because the influence of SHB is overcome by $\bar{\Gamma}_{y}$. In addition, $\omega_{f}$ is increased with $\kappa L$ as well as the total energy stored inside the laser cavity. Hence, the value of $\omega_{f}$ can be enhanced for devices with wide stripe width and high $\kappa L$.

Our analytical analysis on $\omega_{f}$ is also verified by our numerical results. It is found that the combined longitudinal and lateral effects have less influence on the damping rate of the lasers. Hence, the ignorance of the effective gain compression factors due to the combined longitudinal and lateral effects in the derivation of $\omega_{f}$ in Section II-B is satisfied.

In the design of DTWG-DFB lasers, a wide tapered region and high $\kappa L$ are required to enhance $\omega_{f}$ provided that 


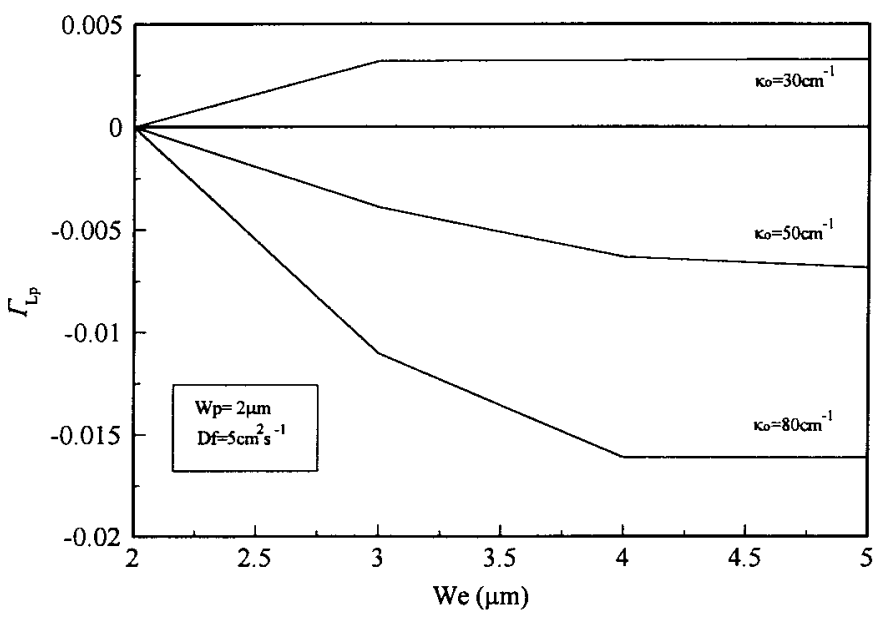

(a)

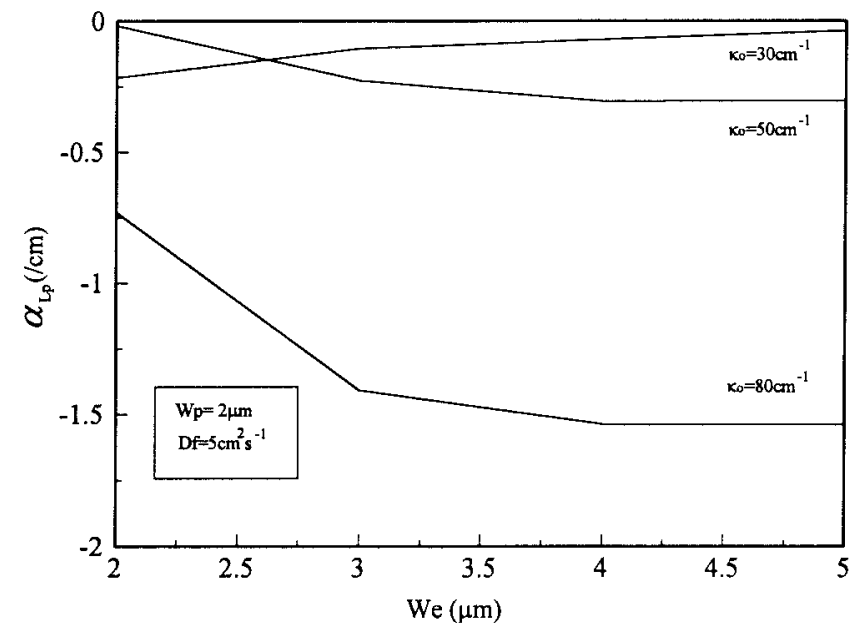

(b)

Fig. 10. The variation of (a) the overlapping integral $\Gamma_{L p}$ and (b) the overlapping integral $\alpha_{L p}$ for devices with $\kappa_{o}=30-80 \mathrm{~cm}^{-1}$ and $W_{e}=$ $2-5 \mu \mathrm{m}$.

single-lateral-mode operation can still be maintained by the diffraction effects of the DTWG structure. Therefore, the DTWG structure gives a simple solution to maintain high $\omega_{f}$ and stable lateral mode operation, and this is the major advantage over the uniform stripe devices. This is because in order to enhance $\omega_{f}$ in uniform stripe BH-DFB lasers, it may require increasing the stripe width (i.e., $\Gamma_{y}$ changes from $\sim 0.7$ to $\sim 1.0$ ) which will also excite high-order lateral modes.

The dynamic behavior of DTWG-DFB lasers is analyzed theoretically. It is shown that the modulation bandwidth DFB lasers, especially for large $\kappa L$, can be enhanced with a DTWG structure. Apart from stable-single-mode and high power characteristics, DTWG-DFB lasers also have significant improvement in modulation speed over the conventional uniform stripe devices.

\section{APPENDIX A}

We assumed the field profiles in the transverse $(x)$ and lateral $(y)$ directions satisfy [7]

$$
\left[\frac{\partial^{2}}{\partial x^{2}}+\varepsilon_{\mathrm{eff}} k_{o}^{2}-\beta_{\mathrm{eff}}^{2}\right] \phi_{o}=0
$$

$$
\left[\frac{\partial^{2}}{\partial y^{2}}+\left(\beta_{\mathrm{eff}}^{2}-\beta^{2}\right)\right] \psi_{\circ}=0
$$

where $\beta_{\text {eff }}$ is the effective propagation coefficient in lateral direction and $\beta$ is a propagation constant to be determined. $\phi_{o}$ and $\psi_{o}$ are the fundamental transverse and lateral field distributions, respectively. $\varepsilon_{f}$ is the effective permittivity and is given by [8]

$$
\begin{aligned}
\varepsilon_{f}(y, z, t) & \\
= & n_{a}^{2}(y)-n_{a}(y) \Gamma_{x} \frac{\alpha_{m} \lambda_{o}}{2 \pi} a_{N}\left(N(y, z, t)-N_{\mathrm{th}}\right) \\
& +i \frac{\lambda_{o} n_{a}(y)}{2 \pi} \Gamma_{x}\left[a_{N}\left(N(y, z, t)-N_{o}\right)-\alpha_{s}\right]
\end{aligned}
$$

where $n_{a}$ is the built-in refractive index of the waveguide, $\alpha_{m}$ is the material linewidth enhancement factor, and $N_{\text {th }}$ is the threshold carrier concentration.

The effective refractive index $n_{\text {eff }}$ given in (3) is defined as [7]

$$
n_{\mathrm{eff}}=\beta_{\mathrm{eff}} / k_{o}
$$

where $k_{o}=2 \pi / \lambda_{o}$. The transverse confinement factor $\Gamma_{x}$ is expressed as

$$
\Gamma_{x}=\int_{\substack{\text { active } \\ \text { layer }}} \phi_{o}^{2} d x
$$

and the lateral confinement factor $\Gamma_{y}$ is given by

$$
\Gamma_{y}=\int_{0}^{w} \psi_{o}^{2} d y
$$

where $w$ is the width of waveguide. The longitudinal coupling coefficient $\kappa$ is given by

$$
\kappa=\frac{1}{2 \beta_{O}} \int_{0}^{w} \int_{\substack{\text { grating } \\ \text { layer }}} k_{o}^{2} A_{-2} \phi_{o}^{2} \psi_{o}^{2} d x d y \approx \kappa_{o} \Gamma_{y}
$$

where $A_{-2}$ is the Fourier coefficient of the dielectric grating, $\beta_{O}$ is the propagation constant at Bragg frequency, and $\kappa_{O}$ is the coupling coefficient of PAR.

The first- and second-order coupling parameters are given by

$$
\begin{aligned}
& \xi_{1}=\int_{-w / 2}^{w / 2} \cos (2 \pi y / w) \psi_{o}^{2}(y) d y \\
& \xi_{2}=\int_{-w / 2}^{w / 2} \cos ^{2}(2 \pi y / w) \psi_{o}^{2}(y) d y
\end{aligned}
$$

\section{APPENDIX B}

The first-, second-, and third-order lateral confinement factor $\Gamma_{y 1}, \Gamma_{y 2}$, and $\Gamma_{y 3}$ are given by

$$
\begin{aligned}
\Gamma_{y 1} & =\frac{1}{L} \int_{0}^{L} \Gamma_{y}(z)(1+f(z)) d z=\bar{\Gamma}_{y}+\Gamma_{L p} \\
\Gamma_{y 2} & =\frac{1}{L} \int_{0}^{L} \Gamma_{y}(z) f(z)(1+f(z)) d z \\
\Gamma_{y 3} & =\frac{1}{L} \int_{0}^{L} \Gamma_{y}(z) f^{2}(z)(1+f(z)) d z
\end{aligned}
$$


where $\bar{\Gamma}_{y}=\frac{1}{L} \int_{0}^{L} \Gamma(z) d z$ is the average value of of $\Gamma_{y}(z)$ and $\Gamma_{L p}=\frac{1}{L} \int_{0}^{L} \Gamma_{y}(z) f(z) d z$ is the overlapping integral of $\Gamma_{y}(z)$ and $f(z)$.

The equivalent cavity loss $\alpha_{p}^{\prime}$ is given by

$$
\alpha_{p}^{\prime}=\frac{1}{L} \int_{0}^{L} \alpha_{p}(z)(1+f(z)) d z=\alpha_{s}+\alpha_{L p}
$$

where $\alpha_{L p}$ is given by

$$
\begin{aligned}
\alpha_{L p} & =\frac{1}{L} \int_{0}^{L} \frac{1}{|F|^{2}+|R|^{2}}\left(\frac{\partial}{\partial z}|F|^{2}-|R|^{2}\right) f(z) d z \\
& =\frac{1}{P_{0} L} \int_{0}^{L} \frac{1}{(1+f(z))}\left(\frac{\partial}{\partial z}|F|^{2}-|R|^{2}\right) f(z) d z
\end{aligned}
$$

The normalization factor $\eta$ is equal to

$$
\eta=\frac{1}{L} \int_{0}^{L} f^{2}(z) d z
$$

\section{REFERENCES}

[1] S. F. Yu, "Quasi 3-dimensional large signal dynamic model of DFB lasers," IEEE J. Quantum Electron., vol. 32, pp. 424-432, Mar. 1996.

[2] _ "Double tapered waveguide distributed feedback lasers for high power single mode operation," IEEE J. Quantum Electron., vol. 33, pp. 71-80, Jan. 1997.
[3] S. F. Yu and E. H. Li, "Influence of lateral field on the relaxation oscillation frequency of semiconductor lasers," IEEE J. Quantum Electron., vol. 32, pp. 1-3, Jan. 1996.

[4] S. F. Yu, R. G. S. Plumb, L. M. Zhang, M. C. Nowell, and J. E. Carroll, "Large signal dynamic behavior of distributed feedback lasers including lateral effects," IEEE J. Quantum Electron., vol. 30, pp. 1740-1750, Aug. 1994.

[5] G. Morthier, F. Libbrecht, K. David, P. Vankwikelberge, and R. G. Baets, "Theoretical investigation of the second-order harmonic distortion in the AM response of $1.55 \mu \mathrm{m}$ F-P and DFB lasers," IEEE J. Quantum Electron., vol. 27, pp. 1990-2002, 1991.

[6] P. Vankwikelberge, F. Buytaert, A. Franchois, R. Baets, P. I. Kuindersma, and C. W. Fredriksz, "Analysis of the carrier-induced FM response of DFB lasers: Theoretical and experimental case studies," IEEE $J$. Quantum Electron., vol. 25, pp. 2239-2254, 1989.

[7] M. J. Adams, An Introduction to Optical Waveguides. New York: Wiley, ch. 6.

[8] S. F. Yu and E. H. Li, "The effects of lateral modes on static and dynamic behavior of buried heterostructure DFB lasers," in Proc. Inst. Elect. Eng., Optoelectronics, vol. 142, pt. J, no. 2, pp. 97-102, 1995.

[9] R. Tucker and D. J. Pope, "Circuit modeling of diffusion on damping in a narrow-stripe semiconductor laser," IEEE J. Quantum Electron., vol. QE-19, pp. 1179-1183, 1983.

S. F. Yu, for photograph and biography, see p. 1008 of the June 1997 issue of this JOURNAL. 